\title{
ARTICLE
}

\section{ACUTE LYMPHOBLASTIC LEUKEMIA}

\section{Hepatic sinusoidal obstruction syndrome and short-term application of 6-thioguanine in pediatric acute lymphoblastic leukemia}

\author{
Martin Stanulla $\mathbb{D}^{1}$ - Elke Schaeffeler ${ }^{2,3}$ - Anja Möricke ${ }^{4}$ Swantje Buchmann ${ }^{4}$ - Martin Zimmermann ${ }^{1}$. \\ Svitlana Igel ${ }^{2} \cdot$ Kjeld Schmiegelow $^{5} \cdot$ Christian Flotho $^{6} \cdot$ Hans Hartmann $^{7} \cdot$ Sabine Illsinger $^{7}$ - Axel Sauerbrey ${ }^{8}$. \\ Stefanie V. Junk ${ }^{1} \cdot$ Peter Schütte $^{1} \cdot$ Laura Hinze $^{1} \cdot$ Melchior Lauten $^{9} \cdot$ Simon Modlich $^{4} \cdot$ Reinhard Kolb $^{10}$.

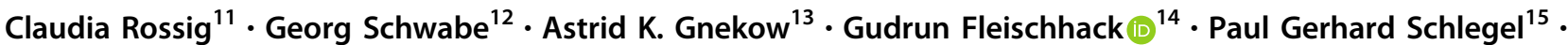 \\ Holger J. Schünemann ${ }^{16} \cdot$ Christian P. Kratz ${ }^{1}$ Gunnar Cario ${ }^{4}$ Martin Schrappe ${ }^{4}$ Matthias Schwab Mch,17 $^{2,1}$
}

Received: 6 October 2020 / Revised: 27 January 2021 / Accepted: 18 February 2021 / Published online: 13 March 2021

(c) The Author(s) 2021. This article is published with open access

\begin{abstract}
Long-term treatment with 6-thioguanine (6-TG) for pediatric acute lymphoblastic leukemia (ALL) is associated with high rates of hepatic sinusoidal obstruction syndrome (SOS). Nevertheless, current treatment continues to use short-term applications of 6-TG with only sparse information on toxicity. 6-TG is metabolized by thiopurine methyltransferase (TPMT) which underlies clinically relevant genetic polymorphism. We analyzed the association between hepatic SOS reported as a serious adverse event (SAE) and short-term 6-TG application in 3983 pediatric ALL patients treated on trial AIEOP-BFM ALL 2000 (derivation cohort) and defined the role of TPMT genotype in this relationship. We identified 17 patients $(0.43 \%)$ with hepatic SOS, 13 of which with short-term exposure to 6-TG $(P<0.0001)$. Eight of the 13 patients were heterozygous for low-activity TPMT variants, resulting in a 22.4 -fold $(95 \%$ confidence interval $7.1-70.7 ; P \leq 0.0001)$ increased risk of hepatic SOS for heterozygotes in comparison to TPMT wild-type patients. Results were supported by independent replication analysis. All patients with hepatic SOS after short-term 6-TG recovered and did not demonstrate residual symptoms. Thus, hepatic SOS is associated with short-term exposure to 6-TG during treatment of pediatric ALL and SOS risk is increased for patients with low-activity TPMT genotypes.
\end{abstract}

\section{Introduction}

Pediatric acute lymphoblastic leukemia (ALL) patients treated according to modern treatment protocols can expect to achieve long-term cure in $\sim 90 \%$ of cases, but still a significant proportion of patients suffers from relapse and therapy-related toxicities [1, 2]. Thus, the challenge to further improve treatment for children with ALL addresses not only cure from leukemia, but also reduction of short-term and long-term therapy-associated toxicities [3]. This can be achieved by an optimized potential realization of risk and subsequent treatment adaptation to avoid unwanted events.

Supplementary information The online version contains supplementary material available at https://doi.org/10.1038/s41375021-01203-7.

Martin Stanulla

stanulla.martin@mh-hannover.de

Extended author information available on the last page of the article
The thiopurines 6-mercaptopurine (6-MP) and 6-thioguanine (6-TG) play an essential role in treatment protocols for ALL. Randomized controlled trials (RCTs) comparing the efficacy and toxicity of 6-MP with 6-TG in interim maintenance and maintenance therapy of childhood ALL have demonstrated dose-dependent high rates $(>10 \%)$ of severe hepatotoxic side effects [4-6]. These side effects have features of sinusoidal obstruction syndrome (SOS) and are associated with long-term exposure to 6-TG. Hepatic SOS after long-term exposure to 6-TG is often reversible, clinical symptoms are commonly less severe, and mortality is very low compared to hepatic SOS in association with hematopoietic stem cell transplantation (HSCT) [6, 7]. Hepatic SOS occurring in association with short-term exposure toward 6-TG has been repeatedly described, but is rare and less well characterized [4, 5, 8-11].

Hepatic SOS, formerly known as veno-occlusive disease (VOD), almost exclusively occurs after exposure to drugs or other toxic stimuli and, in histopathology, 
Fig. 1 Treatment outline of AIEOP-BFM ALL 2000.

Details of treatment elements are listed in Supplementary Table 1.

Results of the randomizations

(R) and data on allogeneic hematopoietic stem cell transplantation (HSCT) have been published before [16, 27]. SR indicates standard risk; MR, intermediate risk; HR, high risk; closed boxes indicate treatment elements: $\mathrm{I}_{\mathrm{A}}$, Protocol I phase A; $\mathrm{D}^{+}$indicates induction with dexamethasone and $\mathrm{P}+$ with prednisone; $\mathrm{I}_{\mathrm{B}}$, Protocol I phase B; M, Protocol M; II, Protocol II; III, Protocol III; HR1', HR2' and $\mathrm{HR}^{\prime}$ are intensive high-risk treatment blocks; 6-MP/MTX, interim maintenance or maintenance therapy with 6mercaptopurine and methotrexate; CR cranial irradiation; closed red boxes indicate the treatment elements in which hepatic SOS cases were observed. The asterisk indicates the second episode of hepatic SOS in patient 16 (Table 2) (color figure online).

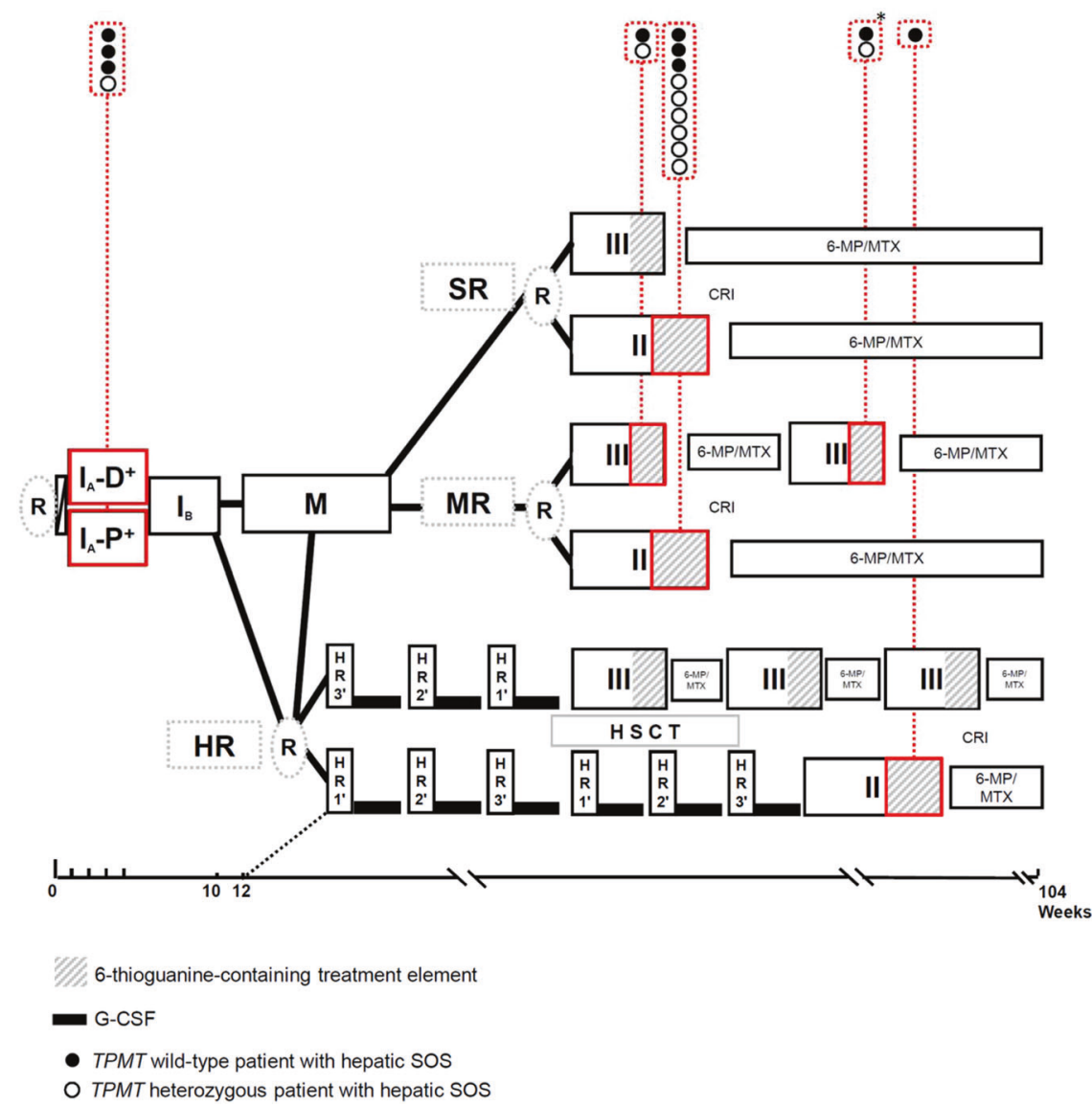

correlates with central obstruction of liver sinusoids, liver cell necrosis and hemorrhage [6, 7]. The clinical signs and symptoms of hepatic SOS include abdominal pain and swelling, portal hypertension with liver enzyme elevations and jaundice [6, 7]. A pharmacokinetic study accompanying a British RCT established a role for the thiopurine detoxifying enzyme thiopurine methyltransferase (TPMT) which underlies phenotypically relevant genetic polymorphism [12, 13] in the association of 6-TG with hepatic SOS [14]. However, the latter relationship could not be confirmed by others [8, 15]. As a consequence of the increased toxicity observed with exposure to 6-TG for treatment of ALL, 6-MP remained the standard thiopurine of choice for maintenance treatment of ALL. Since discontinuation of long-term 6-TG application, the previously associated hepatic complications are only rarely observed during treatment for ALL. Nevertheless, due to its anti-leukemic potency, currently the majority of clinical protocols for ALL continue to use short-term applications of 6-TG during intensification elements. Therefore, we analyzed the reported frequencies and characteristics of hepatic SOS in a large multicenter Berlin-Frankfurt-Münster (BFM) RCT on treatment of pediatric ALL in which 6-TG was applied for 2-week intervals during late-intensification therapy.

\section{Materials/subjects and methods}

\section{Study individuals}

From August 1, 2000, to May 31, 2010, a total of 3983 ALL patients between 1 and 18 years of age were diagnosed in one of the participating study centers in Germany and registered in trial AIEOP-BFM ALL 2000 [16-18] (Supplementary Fig. 1). Treatment in this trial, which we used as derivation cohort, contained standard multidrug chemotherapeutic regimens (for details see Fig. 1 and Supplementary Table 1) and, in parts of the patient population, cranial irradiation and/or HSCT. 6-TG $\left(60 \mathrm{mg} / \mathrm{m}^{2} / \mathrm{d}\right)$ was applied for 2 weeks in the second phase of two lateintensification elements, the so-called Protocol II and Protocol III (Fig. 1, Supplementary Table 1). Out of the 3983 patients, 813 had TPMT genotype information available (Supplementary Fig. 1). Our replication cohort included 1566 patients between 1 and 18 years of age, diagnosed with pediatric ALL from June 1, 2010, to December 31, 2016, and treated in the non-experimental arms of trial AIEOP-BFM ALL 2009 incorporating the lateintensification element Protocol II [19]. Informed consent was obtained from all patients and/or their guardians. Both study protocols AIEOP-BFM ALL 2000 and 
AIEOP-BFM ALL 2009 were approved by the competent ethics committees and trials were registered at http://clinica ltrials.gov (NCT00430118 and NCT01117441).

Cases of hepatic SOS were identified through searching the AIEOP-BFM ALL 2000 and AIEOP-BFM ALL 2009 serious adverse event (SAE) databases. An SAE was defined as any untoward medical occurrence that (1) resulted in death; (2) was life threatening (defined as an event in which the patient was at risk of death at the time of the event; it did not refer to an event which hypothetically might have caused death if it would have been more severe); (3) required or prolonged hospitalization; (4) resulted in persistent or significant disability/incapacity; (5) was a congenital anomaly/birth defect in the offspring. Hepatic SOS was characterized according to the Ponte di Legno (PdL) diagnostic criteria for SOS in children with ALL [3] (Supplementary Table 2) and the European Society for Blood and Marrow Transplantation diagnostic criteria for hepatic SOS/VOD in pediatric patients [7]. Patients developing hepatic SOS in association with HSCT were not included in this study.

\section{TPMT genotyping}

DNA extraction from fresh mononuclear cells was performed as described previously [20]. Genotyping for TPMT was performed by standard RQ-PCR-based genotyping for the variant $T P M T^{*} 2$ and $T P M T^{*} 3$ alleles, which are associated with low TPMT activity [21].

\section{Statistical analysis}

Differences in the distribution of categorical variables were analyzed by chi-squared or Fisher's exact tests. The McNemar's test was used on paired dichotomous data. We estimated the odds ratios (derivative cohort) or relative risks (replication cohort) for and calculated their associated 95\% confidence intervals [22]. We report exact $p$ values. The SPSS (SPSS Inc., Chicago, IL) and SAS (SAS-PC, Version 9.1, Cary, NC: SAS Institute Inc.) statistical packages were used for the analyses.

\section{Results}

We identified 17 patients $(0.43 \%)$ with hepatic SOS reported as an SAE out of 3983 patients in the derivation cohort (AIEOP-BFM ALL 2000) (see Supplementary Fig. 1 for the analytical workflow of this study). No significant differences with regard to the distribution of important clinical characteristics related to pediatric ALL were detected between patients with reported hepatic SOS in comparison to those without this treatment complication (Table 1). Four patients developed hepatic SOS during
Table 1 Characteristics of 17 patients with hepatic sinusoidal obstruction syndrome (SOS) reported as a severe adverse event during treatment for acute lymphoblastic leukemia (ALL) in comparison to the remaining patient population of trial AIEOP-BFM ALL 2000.

\begin{tabular}{|c|c|c|c|}
\hline & $\begin{array}{l}\text { Patients with reported } \\
\text { hepatic SOS } \\
(n=17) \\
n(\%)\end{array}$ & $\begin{array}{l}\text { Patients without } \\
\text { reported hepatic SOS } \\
(n=3966) \\
n(\%)\end{array}$ & ${ }^{\mathrm{a}} P$ \\
\hline \multicolumn{4}{|l|}{ Gender } \\
\hline Male & $12(70.6)$ & $2193(55.3)$ & \\
\hline Female & $5(29.4)$ & $1773(44.7)$ & 0.231 \\
\hline \multicolumn{4}{|c|}{ Age at diagnosis (years) } \\
\hline$<10$ & $14(82.4)$ & $2946(74.3)$ & \\
\hline$\geq 10$ & $3(17.6)$ & $1020(25.7)$ & 0.584 \\
\hline \multicolumn{4}{|l|}{ Initial $\mathrm{WBC}^{\mathrm{b}}\left(10^{9} / \mathrm{l}\right)$} \\
\hline$<50$ & $13(76.5)$ & $3139(79.2)$ & \\
\hline$\geq 50$ & $4(23.5)$ & $825(20.8)$ & 0.766 \\
\hline n.a. & - & $2(0.1)$ & \\
\hline \multicolumn{4}{|l|}{ Immunophenotype } \\
\hline B-cell precursor & $17(100)$ & $3305(85.2)$ & \\
\hline T-cell precursor & - & $555(14.3)$ & \\
\hline other & - & $19(0.5)$ & 0.223 \\
\hline n.a. & - & $87(2.2)$ & \\
\hline \multicolumn{4}{|l|}{ CNS disease ${ }^{\mathrm{C}}$} \\
\hline No & $15(88.2)$ & $3584(90.4)$ & \\
\hline Yes & $1(5.9)$ & $135(3.4)$ & 0.448 \\
\hline n.a. & $1(5.9)$ & $247(6.2)$ & \\
\hline \multicolumn{4}{|c|}{ ETV6-RUNX1 rearrangement } \\
\hline Negative & $13(76.5)$ & $2883(72.7)$ & \\
\hline Positive & $4(23.5)$ & $839(21.2)$ & 0.999 \\
\hline n.a. & - & $244(6.2)$ & \\
\hline \multicolumn{4}{|l|}{ Prednisone response ${ }^{\mathrm{d}}$} \\
\hline Good & $16(94.1)$ & 3539 (89.2) & \\
\hline Poor & $1(5.9)$ & $394(9.9)$ & 0.999 \\
\hline n.a. & - & $33(0.8)$ & \\
\hline \multicolumn{4}{|l|}{ Risk group ${ }^{\mathrm{e}}$} \\
\hline SR & $6(35.3)$ & $1305(32.9)$ & \\
\hline MR & $8(47.1)$ & $2050(51.7)$ & \\
\hline HR & $3(17.6)$ & $609(15.4)$ & \\
\hline Other & - & $2(0.1)$ & 0.845 \\
\hline \multicolumn{4}{|l|}{ TPMT } \\
\hline wild-type & $8(35.3)$ & $755(19.0)$ & \\
\hline Heterozygous & $9(47.1)$ & $54(1.4)$ & \\
\hline Deficient & - & $4(0.1)$ & $<0.0001$ \\
\hline n.a. & - & $3153(79.5)$ & \\
\hline
\end{tabular}

${ }^{\mathrm{a}} P \chi 2$ or Fisher's exact test.

${ }^{\mathrm{b}} W B C$ white blood cell count.

${ }^{\mathrm{c}} \mathrm{CNS}$ positive: puncture nontraumatic, $>5 \mathrm{WBC} / \mu \mathrm{L}$ cerebrospinal fluid with identifiable blasts

${ }^{\mathrm{d}}$ Good: $<1000$ leukemic blood blasts/ $\mu$ l on treatment day 8, poor: $\geq 1000 / \mu \mathrm{l}$.

${ }^{\text {e }}$ Risk group stratification included minimal residual disease (MRD) analysis and required two MRD targets with sensitivities of $\leq 1 \times 10^{-4}$, SR patients were MRD-negative on treatment days 33 and 78 , HR patients had residual disease of $\geq 5 \times 10^{-4}$ on treatment day 78, all the remaining MRD results were stratified into the MR group, further HR criteria were prednisone poor response or $\geq 5 \%$ leukemic blasts in the bone marrow on day 33 or positivity for $t(4 ; 11)$ or its molecular equivalent (MLL-AF4 gene fusion); were stratified into the high-risk group independent of their MRD results. 
induction treatment with a glucocorticoid, vincristine, L-asparaginase, daunorubicin, and intrathecal methotrexate, two of them in association with either acute pancreatitis or severe sepsis (Fig. 1 and Table 2; for treatment details see Supplementary Table 1). In 13 patients, hepatic SOS strongly clustered with short-term exposure to 6-TG during the second phase of late-intensification elements-ten patients were observed in late-intensification Protocol II and three patients during Protocol III (Fig. 1 and Table 2). One patient developed a second episode of hepatic SOS after rechallenge with 6-TG in a second application of Protocol III. The difference of frequencies of hepatic SOS in 6-TGcontaining elements compared to other treatment elements was highly significant $(P \leq 0.0001)$. All cases associated with 6-TG exposure were of moderate grade according to PdL criteria [3]. With one exception, all patients received defibrotide (Table 2). All patients with 6-TG-associated hepatic SOS recovered, did not demonstrate residual symptoms such as portal hypertension/splenomegaly or nodular regenerative hyperplasia on follow-up, and are in continuous complete remission (Table 2).

The numbers of patients exposed to 6-TG in trial AIEOPBFM ALL 2000 were 2531 for Protocol II and 1212 for Protocol III. This results in a rate of hepatic SOS in association with 6-TG of $0.40 \%$ in Protocol II compared to $0.25 \%$ in Protocol III $(P=0.47)$. Co-medication with 6-TG in these two late-intensification elements consisted of similar applications of cytarabine and intrathecal methotrexate, while a single cyclophosphamide application was given at $1000 \mathrm{mg} / \mathrm{m}^{2}$ in Protocol II compared to $500 \mathrm{mg} / \mathrm{m}^{2}$ in Protocol III (Supplementary Table 1). Thus, we could not evaluate if cyclophosphamide modifies the risk of hepatic SOS in association with 6-TG treatment.

Next, we compared a representative subset of 813 AIEOP-BFM ALL 2000 patients [20] with available TPMT genotype data and no reported history of hepatic SOS to the 13 patients with hepatic SOS under exposure to 6-TG during late-intensification treatment (Protocol II/Protocol III). Eight (61.5\%) of the 13 patients with hepatic SOS were heterozygous for TPMT variants conferring low enzyme activity compared to $54(6.6 \%)$ in the cohort without reported hepatic SOS (Table 1 and Fig. 1). In contrast, only one out the four patients with hepatic SOS during induction treatment carried a low-activity TPMT allele (Table 2). The odds ratio for hepatic SOS under exposure to 6-TG was 22.4 (95\% confidence interval 7.1-70.7; $P \leq 0.0001$ ) for TPMT heterozygotes in comparison to TPMT wild-type patients. To replicate this association, we analyzed 1566 patients treated on the non-experimental arm of trial AIEOP-BFM ALL 2009. These patients received the same 6-TG containing Protocol II as given in the previous trial AIEOP-BFM ALL 2000. A search of the AIEOP-BFM ALL 2009 SAE database for study individuals with reported hepatic SOS associated with Protocoll II identified nine out of 1566 ALL patients (0.57\%) (Supplementary Table 3). Three of the nine patients were heterozygous for TPMT alleles conferring low enzyme activity (all $T P M T * 1 / * 3 A$ ) resulting in a 6.73 -fold increased relative risk $(95 \%$ confidence interval $1.71-26.53 ; P=0.007$ ) of hepatic SOS in association with 6-TG for heterozygotes in comparison to TPMT wild-type patients.

\section{Discussion}

In the present analysis, short-term exposure to 6-TG during treatment for pediatric ALL was associated with hepatic SOS and patients with TPMT genotypes conferring low enzyme activity were at increased risk. Supporting the specificity of the association of hepatic SOS with 6-TG exposure, no patient with hepatic SOS in association with the second phase of Protocol I was detected. This treatment element equals the second phases of Protocol II and Protocol III except that 6MP is applied instead of 6-TG (Supplementary Table 1). Therefore, in a similar treatment context, hepatic SOS recorded as an SAE was only observed in context with application of 6-TG, but virtually absent with 6-MP. The latter observation suggests that replacement of 6-TG by 6-MP in lateintensification elements may abolish or at least significantly reduce the appearance of hepatic SOS during lateintensification. Due to the currently observed good longterm outcome of our patients with hepatic SOS in association with short-term 6-TG and the lack of data on equivalent antileukemic efficacy of 6-MP versus 6-TG in late-intensification elements we did not yet change the drug compositions of Protocols II and III. However, we alert clinicians to the specific risk of hepatic SOS under exposure to 6-TG in our clinical protocols. A potential explanation for the differential impact of 6-TG and 6-MP on risk of hepatic SOS may be specific angioprotective properties attributed to 6-MP. In different vascular disease model systems, 6-MP was shown to impact on the expression of adhesion molecules, to reduce monocyte attraction/adhesion, to induce monocyte apoptosis, to inhibit re-stenosis and to attenuate inflammation, while stimulating endothelial coverage [23-26].

In a single institution study, McAtee et al. recently published a series of ten $(1.5 \%)$ hepatic SOS out of 680 pediatric ALL patients treated at Texas Children's Cancer Center [9]. Compared to long-term exposure during 6-TGbased maintenance treatment of ALL, hepatic SOS in context of short-term 6-TG exposure was associated with earlier onset and higher mortality (20\%) which led the authors to conclude that hepatic SOS after short-term 6-TG treatment may be a clinically distinct phenotype with similarities to hepatic SOS in association with HSCT. Regarding timing of hepatic SOS our data confirm the 


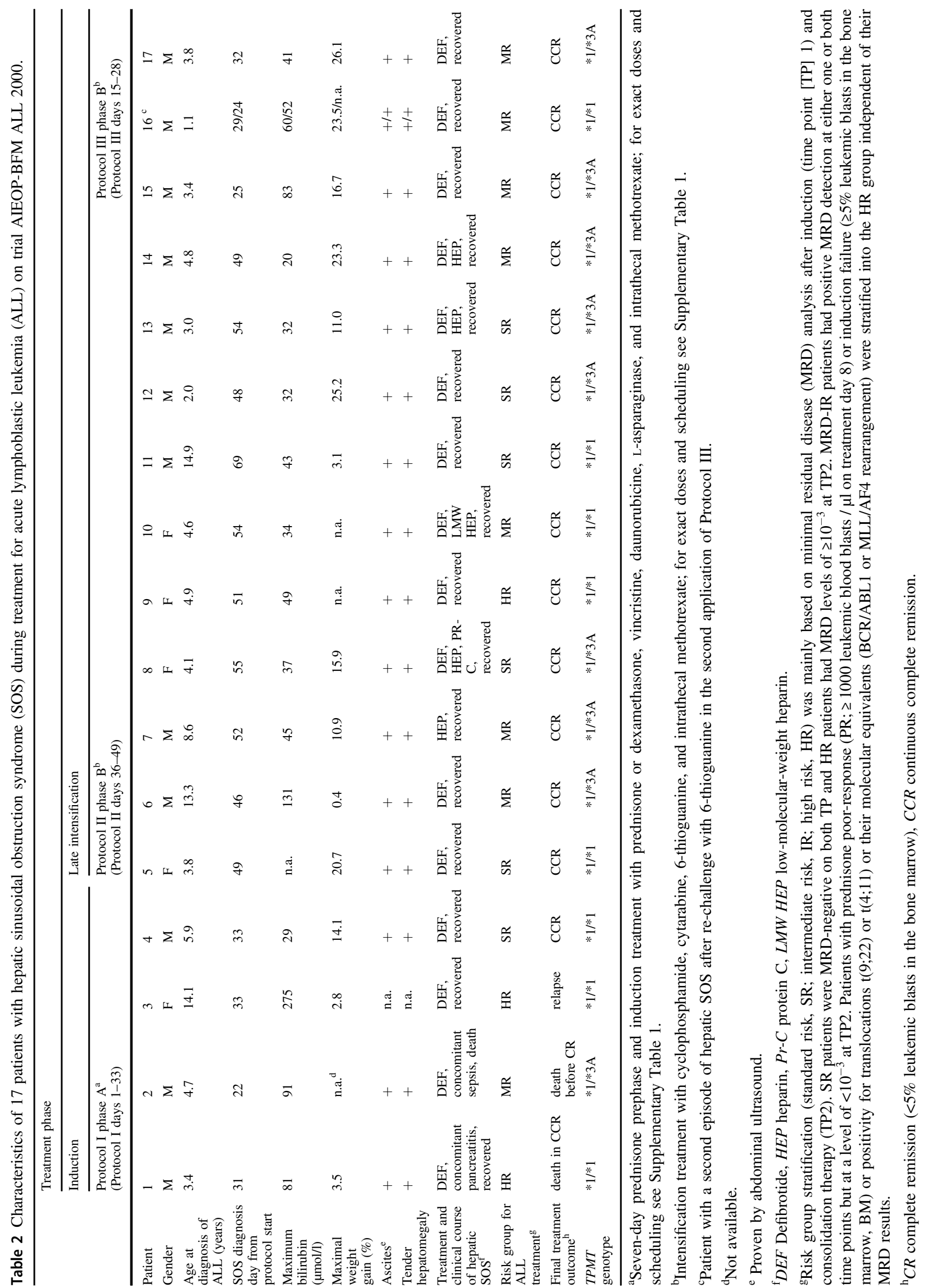


observation of McAtee et al. of an early onset in close association with 6-TG application (Table 2) [9]. Similarly, we also observed a high frequency of low TPMT activity in our patients. This may support the hypothesis that altered 6TG metabolism may either modify the severity of hepatic SOS or-as associations with long-term exposure are contradictory-reflect a stronger effect of variant TPMT in association with short-term exposure during late intensification. In addition, our data provide a further instructive example for the importance to subject pediatric ALL patients treated with thiopurines to TPMT genotyping. Intriguingly, McAtee et al. described confirmed or suspected infections prior to diagnosis of hepatic SOS in six out of ten cases with hepatic SOS [9]. Due to unavailability of data, we cannot reliably evaluate this association in our study. The single patient in our study suffering from concomitant sepsis who finally died in association with hepatic SOS was observed during induction treatment and not in association with 6-TG. Although also potential differences in protocol-specific treatment exposures may have contributed here, the lower frequency of hepatic SOS in our study compared to McAtee's study is likely due to the inclusion of hepatic SOS reported as an SAE, only. This indicates that we probably did not capture all/less severe episodes of hepatic SOS. Therefore, careful monitoring for signs of hepatic SOS in association with short-term 6-TG exposure in future studies will have to characterize the entire clinical spectrum and determine the true incidence of this complication.

We conclude that hepatic SOS reported as an SAE on contemporary ALL-BFM trials cluster with short-term application of 6-TG during late-intensification elements and are associated with TPMT genotype. Particularly for TPMT heterozygotes, replacement of 6-TG by 6-MP may be an effective measure to reduce the incidence of hepatic SOS during treatment for pediatric ALL.

Acknowledgements This article is dedicated to our late dear friend and colleague Professor Giuseppe (Beppe) Basso from Padova - an outstanding pediatric hemato-oncologist and passionate leukemia researcher. We are indebted to all patients, parents, nurses, and doctors participating in/contributing to AIEOP-BFM ALL 2000 and AIEOP-BFM ALL 2009. This work was funded by the European Commission (FP7, TRANSCALL 2), the Robert-Bosch-Stiftung, Stuttgart, the Deutsche Forschungsgemeinschaft (DFG, German Research Foundation) under Germany's Excellence Strategy-EXC 2180-390900677, the Madeleine-Schickedanz-Kinderkrebsstiftung, Verein für krebskranke Kinder Hannover e.V., and the Deutsche Krebshilfe.

Funding Open Access funding enabled and organized by Projekt DEAL.

\section{Compliance with ethical standards}

Conflict of interest The authors declare no competing interests.
Publisher's note Springer Nature remains neutral with regard to jurisdictional claims in published maps and institutional affiliations.

Open Access This article is licensed under a Creative Commons Attribution 4.0 International License, which permits use, sharing, adaptation, distribution and reproduction in any medium or format, as long as you give appropriate credit to the original author(s) and the source, provide a link to the Creative Commons license, and indicate if changes were made. The images or other third party material in this article are included in the article's Creative Commons license, unless indicated otherwise in a credit line to the material. If material is not included in the article's Creative Commons license and your intended use is not permitted by statutory regulation or exceeds the permitted use, you will need to obtain permission directly from the copyright holder. To view a copy of this license, visit http://creativecommons. org/licenses/by/4.0/.

\section{References}

1. Reedijk AMJ, Coebergh JWW, de Groot-Kruseman HA, van der Sluis IM, Kremer LC, Karim-Kos HE, et al. Progress against childhood and adolescent acute lymphoblastic leukaemia in the Netherlands, 1990-2015. Leukemia. 2020. https://doi.org/10. 1038/s41375-020-01024-0.

2. Vrooman LM, Silverman LB. Treatment of childhood acute lymphoblastic leukemia: prognostic factors and clinical advances. Curr Hematol Malig Rep. 2016;11:385-94.

3. Schmiegelow K, Attarbaschi A, Barzilai S, Escherich G, Frandsen TL, Halsey C, et al. Consensus definitions of 14 severe acute toxic effects for childhood lymphoblastic leukaemia treatment: a Delphi consensus. Lancet Oncol. 2016;17:e231-e239.

4. Vora A, Mitchell CD, Lennard L, Eden TO, Kinsey SE, Lilleyman $\mathrm{J}$, et al. Toxicity and efficacy of 6-thioguanine versus 6mercaptopurine in childhood lymphoblastic leukaemia: a randomised trial. Lancet. 2006;368:1339-48.

5. Stork LC, Matloub Y, Broxson E, La M, Yanofsky R, Sather H, et al. Oral 6-mercaptopurine versus oral 6-thioguanine and venoocclusive disease in children with standard-risk acute lymphoblastic leukemia: report of the Children's Oncology Group CCG1952 clinical trial. Blood. 2010;115:2740-8.

6. Toksvang LN, Schmidt MS, Arup S, Larsen RH, Frandsen TL, Schmiegelow $\mathrm{K}$, et al. Hepatotoxicity during 6-thioguanine treatment in inflammatory bowel disease and childhood acute lymphoblastic leukaemia: a systematic review. PLoS ONE. 2019;14:e212157.

7. Corbacioglu S, Carreras E, Ansari M, Balduzzi A, Cesaro S, Dalle $\mathrm{JH}$, et al. Diagnosis and severity criteria for sinusoidal obstruction syndrome/veno-occlusive disease in pediatric patients: a new classification from the European society for blood and marrow transplantation. Bone Marrow Transplant. 2018;53:138-45.

8. Jacobs SS, Stork LC, Bostrom BC, Hutchinson R, Holcenberg J, Reaman GH, et al. Substitution of oral and intravenous thioguanine for mercaptopurine in a treatment regimen for children with standard risk acute lymphoblastic leukemia: a collaborative Children's Oncology Group/National Cancer Institute pilot trial (CCG-1942). Pediatr Blood Cancer. 2007;49:250-5.

9. McAtee CL, Schneller N, Brackett J, Bernhardt MB, Schafer ES. Treatment-related sinusoidal obstruction syndrome in children with de novo acute lymphoblastic leukemia during intensification. Cancer Chemother Pharmacol. 2017;80:1261-4.

10. Toksvang LN, De Pietri S, Nielsen SN, Nersting J, Albertsen BK, Wehner PS, et al. Hepatic sinusoidal obstruction syndrome during maintenance therapy of childhood acute lymphoblastic leukemia is associated with continuous asparaginase therapy and mercaptopurine metabolites. Pediatr Blood Cancer. 2017;64:e26519. 
11. Pawlik-Gwozdecka D, Irga-Jaworska N, Tomaszewski M, Adamkiewicz-Drożyńska E. Sinusoidal obstruction syndrome in a paediatric patient with acute lymphoblastic leukaemia after completion of reinduction therapy according to ALL Intercontinental Berlin-Frankfurt-Münster 2009. Contemp Oncol. 2018;22:266-9.

12. Evans WE, Hon YY, Bomgaars L, Coutre S, Holdsworth M, Janco R, et al. Preponderance of thiopurine S-methyltransferase deficiency and heterozygosity among patients intolerant to mercaptopurine or azathioprine. J Clin Oncol. 2001;19:2293-301.

13. McLeod HL, Krynetski EY, Relling MV, Evans WE. Genetic polymorphism of thiopurine methyltransferase and its clinical relevance for childhood acute lymphoblastic leukemia. Leukemia. 2000;14:567-72.

14. Lennard L, Richards S, Cartwright CS, Mitchell C, Lilleyman JS, Vora A. The thiopurine methyltransferase genetic polymorphism is associated with thioguanine-related veno-occlusive disease of the liver in children with acute lymphoblastic leukemia. Clin Pharmacol Ther. 2006;80:375-83.

15. Wray L, Vujkovic M, McWilliams T, Cannon S, Devidas M, Stork L, et al. TPMT and MTHFR genotype is not associated with altered risk of thioguanine-related sinusoidal obstruction syndrome in pediatric acute lymphoblastic leukemia: a report from the Children's Oncology Group. Pediatr Blood Cancer. 2014;61:2086-8.

16. Möricke A, Zimmermann M, Valsecchi MG, Stanulla M, Biondi A, Mann G, et al. Dexamethasone vs prednisone in induction treatment of pediatric ALL: results of the randomized trial AIEOP-BFM ALL 2000. Blood. 2016;127:2101-12.

17. Zaliova M, Zimmermannova O, Dörge P, Eckert C, Möricke A, Zimmermann M, et al. ERG deletion is associated with $\mathrm{CD} 2$ and attenuates the negative impact of IKZF1 deletion in childhood acute lymphoblastic leukemia. Leukemia. 2014;28:182-5.

18. Stanulla M, Dagdan E, Zaliova M, Möricke A, Palmi C, Cazzaniga $\mathrm{G}$, et al. IKZF $1^{\text {plus }}$ defines a new minimal residual disease-dependent very-poor prognostic profile in pediatric B-cell precursor acute lymphoblastic leukemia. J Clin Oncol. 2018;36:1240-9.
19. Rizzari C, Lanvers-Kaminsky C, Valsecchi MG, Ballerini A, Matteo C, Gerss J, et al. Asparagine levels in the cerebrospinal fluid of children with acute lymphoblastic leukemia treated with pegylated-asparaginase in the induction phase of the AIEOP-BFM ALL 2009 study. Haematologica. 2019;104:1812-21.

20. Stanulla M, Schaeffeler E, Flohr T, Cario G, Schrauder A, Zimmermann M, et al. Thiopurine methyltransferase (TPMT) genotype and early treatment response to mercaptopurine in childhood acute lymphoblastic leukemia. JAMA. 2005;293:1485-9.

21. Tamm R, Mägi R, Tremmel R, Winter S, Mihailov E, Smid A, et al. Polymorphic variation in TPMT is the principal determinant of TPMT phenotype: a meta-analysis of three genome-wide association studies. Clin Pharmacol Ther. 2017;101:684-95.

22. Altman DG. Practical statistics for medical research. London: Chapman and Hall; 1991.

23. Yoo YG, Na TY, Yang WK, Kim HJ, Lee IK, Kong G, et al. 6Mercaptopurine, an activator of Nur77, enhances transcriptional activity of HIF-1alpha resulting in new vessel formation. Oncogene. 2007;26:3823-34.

24. Pires NM, Pols TW, de Vries MR, van Tiel CM, Bonta PI, Vos M, et al. Activation of nuclear receptor Nur77 by 6-mercaptopurine protects against neointima formation. Circulation. 2007;115:493-500.

25. Pols TW, Bonta PI, Pires NM, Otermin I, Vos M, de Vries MR, et al. 6-Mercaptopurine inhibits atherosclerosis in apolipoprotein $\mathrm{E}^{*} 3$-Leiden transgenic mice through atheroprotective actions on monocytes and macrophages. Arterioscler Thromb Vasc Biol. 2010;30:1591-7.

26. Ruiter MS, van Tiel CM, Doornbos A, Marinković G, Strang AC, Attevelt NJ, et al. Stents eluting 6-mercaptopurine reduce neointima formation and inflammation while enhancing strut coverage in rabbits. PLoS ONE. 2015;10:e138459.

27. Peters C, Schrappe M, von Stackelberg A, Schrauder A, Bader P, Ebell W, et al. Stem-cell transplantation in children with acute lymphoblastic leukemia: a prospective international multicenter trial comparing sibling donors with matched unrelated donors-The ALL-SCT-BFM-2003 trial. J Clin Oncol. 2015;33:1265-74.

\section{Affiliations}

\section{Martin Stanulla $\mathbb{D}^{1} \cdot$ Elke Schaeffeler ${ }^{2,3}$ - Anja Möricke ${ }^{4}$ Swantje Buchmann ${ }^{4}$ - Martin Zimmermann ${ }^{1}$. Svitlana Igel ${ }^{2} \cdot$ Kjeld Schmiegelow $^{5} \cdot$ Christian Flotho $^{6} \cdot$ Hans Hartmann $^{7} \cdot$ Sabine Illsinger $^{7} \cdot$ Axel Sauerbrey $^{8}$. Stefanie V. Junk ${ }^{1} \cdot$ Peter Schütte $^{1} \cdot$ Laura Hinze $^{1} \cdot$ Melchior Lauten $^{9} \cdot$ Simon Modlich $^{4} \cdot$ Reinhard Kolb $^{10}$.

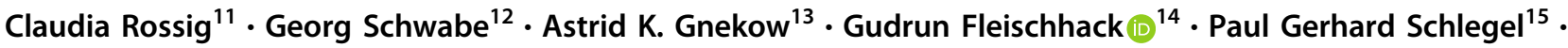 Holger J. Schünemann ${ }^{16}$. Christian P. Kratz ${ }^{1}$ Gunnar Cario ${ }^{4}$ Martin Schrappe ${ }^{4}$ Matthias Schwab Mch,17 $^{2,14}$}

1 Pediatric Hematology and Oncology, Hannover Medical School, Hannover, Germany

2 Dr. Margarete-Fischer-Bosch Institute of Clinical Pharmacology, Stuttgart, Germany

3 Cluster of Excellence iFIT (EXC2180) "Image-guided and Functionally Instructed Tumor Therapies", University of Tübingen, Tübingen, Germany

4 Department of Pediatrics, University Hospital Schleswig-Holstein, Kiel, Germany

5 Department of Pediatrics and Adolescent Medicine, University
Hospital Rigshospitalet, Copenhagen, Denmark

6 Department of Pediatric Hematology and Oncology, University Hospital Freiburg, Freiburg, Germany

7 Department of Pediatric Kidney, Liver, and Metabolic Diseases, Hannover Medical School, Hannover, Germany

8 Pediatric Clinics, Helios Hospital, Erfurt, Germany

9 Department of Pediatrics, University Hospital Schleswig-Holstein, Lübeck, Germany

10 University Children's Hospital, Oldenburg, Germany 
11 Pediatric Hematology and Oncology, University Children's Hospital Münster, Münster, Germany

12 Carl Thiem Hospital, Pediatric Clinics, Cottbus, Germany

13 Pediatric Clinics, University Hospital Augsburg, Augsburg, Germany

14 Pediatrics III, Pediatric Hematology and Oncology, University Hospital Essen, Essen, Germany
15 Pediatric Hematology and Oncology and Stem Cell Transplantation, University Hospital Würzburg, Würzburg, Germany

16 Departments of Health Research Methods, Evidence, and Impact and of Medicine, McMaster University, Hamilton, ON, Canada

17 Departments of Clinical Pharmacology, and of Pharmacy and Biochemistry, University of Tübingen, Tübingen, Germany 